\title{
Antimicrobial resistance profile and multidrug resistance patterns of Streptococcus pneumoniae isolates from patients suspected of pneumococcal infections in Ethiopia
}

\section{BEKELE SHAREW ( $\nabla$ bsharew@gmail.com )}

University of Gondar

\section{Feleke Moges}

University of Gondar College of Medicine and Health Sciences

\section{Gizachew Yismaw}

University of Gondar College of Medicine and Health Sciences

\section{Wondiwossen Abebe}

University of Gondar College of Medicine and Health Sciences

\section{Surafal Fentaw}

Ethiopian Public Health Institute

\section{Didrik Vestrheim}

Norwegian Institute of Public Health: Folkehelseinstituttet

\section{Belay Tessema}

University of Gondar College of Medicine and Health Sciences

\section{Research}

Keywords: Antimicrobial resistance, Multidrug resistance, Streptococcus pneumoniae

Posted Date: December 4th, 2020

DOl: https://doi.org/10.21203/rs.3.rs-120488/v1

License: (9) (i) This work is licensed under a Creative Commons Attribution 4.0 International License. Read Full License

Version of Record: A version of this preprint was published at Annals of Clinical Microbiology and Antimicrobials on April 20th, 2021. See the published version at https://doi.org/10.1186/s12941-02100432-z. 


\section{Abstract \\ Background}

Streptococcus pneumoniae is one of the leading causes of bacterial meningitis and pneumonia in elderly people and children. Antimicrobial-resistant strains of Streptococcus pneumoniae have been detected in all parts of the world and have become one of the greatest challenges to global public health today. The aim of this study is, therefore, to assess the antimicrobial resistance profiles and multidrug resistance patterns of $S$. pneumoniae isolates from patients suspected of pneumococcal infections in Ethiopia.

\section{Methods}

A hospital-based prospective study was conducted from 2018 to 2019 at Addis Ababa and Amhara region referral hospitals. Antimicrobial resistance tests were performed on 57 isolates of S. pneumoniae that were collected from pediatric and adult patients. Samples (cerebrospinal fluid, blood, sputum, eye discharge, ear discharge, and pleural and peritoneal fluids) from all collection sites were initially cultured on $5 \%$ sheep blood agar plates and incubated overnight at 370C in a 5\% CO2 atmosphere. S. pneumoniae was identified and confirmed by typical colony morphology, alpha-hemolysis, Gram staining, optochin susceptibility, and bile solubility test. Drug resistance testing was performed using the E-test method according to recommendations of the Clinical and Laboratory Standards Institute.

\section{Results}

Of the 57 isolates, $17.5 \%$ were fully resistant to penicillin. The corresponding value for both cefotaxime and ceftriaxone was $1.8 \%$. Resistance rates to erythromycin, clindamycin, tetracycline, chloramphenicol and trimethoprim-sulfamethoxazole were $59.6 \%, 17.5 \%, 38.6 \%, 17.5 \%$ and $24.6 \%$, respectively. Multidrug resistance (MDR) was seen in $33.3 \%$ isolates. The most common pattern was co-resistance to penicillin, erythromycin, clindamycin, and tetracycline.

\section{Conclusions}

Most bacterial isolates were susceptible to Ceftriaxone and Cefotaxime. Penicillin has been used as a drug of choice for treating S. pneumoniae infection. However, antimicrobial resistance including multidrug resistance was observed to several commonly used antibiotics including penicillin. Hence, it is important to periodically monitor the antibiotic resistance patterns to choose empirical treatments for better management of pneumococcal infection.

\section{Introduction}


Streptococcus pneumoniae is one of the leading causes of bacterial infections, ranging from self-limiting respiratory tract infections to severe invasive infections. It is a major public health concern, being responsible for an estimated 3.7 million episodes (2.7 million to 4.3 million) in children globally and approximately $50 \%$ of all pneumococcal deaths in 2015 occurred in four countries in Africa and Asia [1].

Antimicrobial resistance has been detected in all parts of the world; it is one of the greatest challenges to global public health today. The problem is increasing resistance to commonly used antimicrobial drugs which have elevated multidrug resistance (MDR). The fight against pneumococcal infections is based on curative treatment with antibiotics and preventive treatment using vaccination [2]. However, the emergence of resistant strains globally poses therapeutic problems. Nearly $40 \%$ of strains are resistant to penicillin, and penicillin resistance often correlates with resistance to other additional antibiotics such as macrolides, tetracyclines, etc. $[3,4]$.

In Asia, an alarming prevalence of penicillin and erythromycin-resistant $S$. pneumoniae has been described [5]. In 1995, an increase in the prevalence of resistance to penicillins, extended-spectrum cephalosporins, trimethoprim-sulfamethoxazole, and macrolides as well as MDR began to be recognized in Taiwan [6]. In Southern Finland, the proportion of MDR pneumococci doubled from 2007 to 2008, before the introduction of the pneumococcal conjugate vaccine (PCV), when it reached 3.6\% [7].

Data on antimicrobial resistance from the African region are limited, but high-level penicillin-resistant $S$. pneumoniae have been described in central Africa [8]. The increasing trend of $S$. pneumoniae antimicrobial-resistance and the emergence of MDR isolates, which may result from inappropriate use of antibiotics and high level of antibiotic use is probably the main factor driving the emergence of resistance worldwide $[9,10]$.

The present study aimed to investigate the antimicrobial resistance profile and multidrug resistance patterns of $S$. pneumoniae isolated from patients suspected of pneumococcal infections in Ethiopia using an E-test, where minimum inhibitory concentrations (MICs) determination is not routinely available.

\section{Methods}

\section{Study area, design, and period}

A hospital-based prospective study was conducted between January 2018 to 2019 at Addis Ababa (Yekatite12 hospital, Alert hospital, and International clinical private laboratory) and Amhara region (University of Gondar comprehensive specialized hospital, Felege Hiwot comprehensive specialized hospital, and Dessie regional laboratory) referral hospitals, Ethiopia. During the study period, 70 phenotypic confirmed S. pneumoniae bacteria were isolated from pediatric and adult patients suspected of pneumococcal infections. All isolates were identified by whole-genome sequencing (WGS). Based on the WGS result, $57(81.4 \%)$ isolates were further confirmed as $S$. pneumoniae. The clinical sources of isolates were cerebrospinal fluid (CSF) $36.4 \%(n=20)$, blood $20 \%(n=12)$, eye discharge $18.2 \%(n=10)$, sputum 15.8\% $(n=10)$, pleural fluid 3.5\% $(n=2)$, ear discharge 3.5\% $(n=2)$ and peritoneal fluid $1.7 \%(n=1)$. 


\section{Isolation of Streptococcus pneumoniae}

As part of routine service, samples (CSF, blood, sputum, eye discharge, ear discharge, and other body fluids) were initially cultured on $5 \%$ sheep blood agar plates. All growing isolates were stored at $-20^{\circ} \mathrm{C}$ and shipped to the University of Gondar Microbiology Laboratory for identification. All isolates received from the collection site were frozen at $-80^{\circ} \mathrm{C}$ until further testing. Isolates were recovered by plating onto $5 \%$ sheep blood and incubated overnight at $37^{\circ} \mathrm{C}$ in a $5 \% \mathrm{CO}_{2}$ atmosphere. S. pneumoniae was identified and confirmed by typical colony morphology, alpha-hemolysis, Gram staining, optochin susceptibility, and bile solubility test. After confirmation, the isolates were stocked in Skimmed Milk-Trypticase Soy-GlucoseGlycerol (STGG) medium and preserved at $-80^{\circ} \mathrm{C}$.

\section{Streptococcus pneumoniae antimicrobial susceptibility testing}

The strains were shipped in dry ice to the Norwegian Reference laboratory for pneumococci at the Norwegian Institute of Public Health where antimicrobial susceptibility testing was performed. Minimum inhibitory concentrations (MICs) were determined by E-test on Mueller-Hinton agar supplemented with $5 \%$ sheep blood by the recommendation of the manufacturer (Biomerieux ${ }^{\circledR}$ ). The antimicrobials tested were penicillin G, cefotaxime, ceftriaxone, erythromycin, clindamycin, tetracycline, chloramphenicol, trimethoprim/ sulfamethoxazole, norfloxacin, and oxacillin. The Clinical and Laboratory Standards Institute (CLSI) 2020 clinical breakpoints for S. pneumoniae were applied to categorize isolates as susceptible, intermediate, or resistant [11]. The CLSI states that the 1- $\mu \mathrm{g}$ oxacillin disk diffusion test is an effective screening method commonly used in clinical laboratories for the detection of penicillin-resistant pneumococci.

The CLSI breakpoints for penicillin, cefotaxime, and ceftriaxone, differ between meningeal and nonmeningeal infections. For penicillin, the breakpoints for meningitis is susceptible $\leq 0.06 \mu \mathrm{g} / \mathrm{ml}$ and resistant $\geq 0.12 \mu \mathrm{g} / \mathrm{ml}$, and for non-meningitis $\leq 2 \mu \mathrm{g} / \mathrm{ml}$ susceptible, $4 \mu \mathrm{g} / \mathrm{ml}$ intermediate and $\geq 8 \mu \mathrm{g} / \mathrm{ml}$ resistance. For Cefotaxime and ceftriaxone, the non-meningitis breakpoint was used to classify isolates as susceptible (MIC $\leq 1 \mu \mathrm{g} / \mathrm{ml})$, intermediate $(\mathrm{MIC}=2 \mu \mathrm{g} / \mathrm{ml})$ or resistant $(\mathrm{MIC} \geq 4 \mu \mathrm{g} / \mathrm{ml})$ and for meningitis susceptible $(\mathrm{MIC} \leq 0.5 \mu \mathrm{g} / \mathrm{ml})$, intermediate $(\mathrm{MIC}=1 \mu \mathrm{g} / \mathrm{ml})$ or resistant $(\mathrm{MIC} \geq 2 \mu \mathrm{g} / \mathrm{ml})$.

Interpretive criteria for norfloxacin are not given by the CLSI 2020. Thus, we used the breakpoints for norfloxacin obtained from the European Committee on Antimicrobial Susceptibility Testing (EUCAST) [12]. Norfloxacin disk diffusion test is an effective screening method for the detection of Moxifloxacin and Levofloxacin resistant pneumococci. MDR was defined as resistance to three or more antimicrobial agents. S. pneumoniae ATCC 49619 was used as the quality control strain and was included in each set of tests to ensure accurate results.

\section{Data analysis}

Data were entered and analyzed using the Statistical Package for the Social Science (version 20; SPSS Inc, Chicago, IL, USA). Discrete variables were expressed as percentages and proportions. 


\section{Results}

Of the 57 patients positive for S. pneumoniae, $33(57.9 \%)$ were males. The age of the patients ranged from 38 days to 71 years; 37 (67.9\%) were children less than 18 years old and 20 (35.1\%) were adults. Among 57 S. pneumoniae strains recovered from different clinical specimens, the most common clinical sources were $\operatorname{CSF}(N=20)$, blood $(N=12)$, sputum $(N=10)$, eye discharge $(N=10)$, ear discharge $(N=2)$, peritoneal $(\mathrm{N}=1)$ and pleural $(\mathrm{N}=2)$. Thus, $35(61.4 \%)$ of the isolates were from cases of invasive pneumococcal disease (IPD), recovered from a sterile body site (CSF, blood, peritoneal and pleural fluids) and $22(38.6 \%)$ were non-IPD strains, recovered from sputum, eye discharge and ear discharge (Table 1)

Table 1: Patient characteristics and isolation of pneumococci at Addis Ababa and Amhara region referral hospitals, Ethiopia, 2018-2019.

\begin{tabular}{|c|c|c|c|}
\hline \multirow[b]{2}{*}{ Characteristics } & \multicolumn{3}{|c|}{ Pneumococcal isolates according to clinical significance } \\
\hline & IPD $(\mathrm{N}=35)$ & Non-IPD $(\mathrm{N}=22)$ & Total $(\mathrm{N}=57)$ \\
\hline \multicolumn{4}{|l|}{ Gender } \\
\hline Male & $24(68.6)$ & $9(40.9)$ & $33(57.9)$ \\
\hline Female & $11(31.4)$ & $13(59.1)$ & $24(42.1)$ \\
\hline \multicolumn{4}{|l|}{ Age (years) } \\
\hline$\leq 18$ & $23(65.7)$ & $14(63.6)$ & $37(64.9)$ \\
\hline$>18$ & $12(34.3)$ & $8(36.4)$ & $20(35.1)$ \\
\hline \multicolumn{4}{|l|}{ Residence } \\
\hline Urban & $23(65.7)$ & $9(40.9)$ & $42(73.7)$ \\
\hline Rural & $12(34.3)$ & $13(59.1)$ & $15(26.3)$ \\
\hline \multicolumn{4}{|l|}{ Data collection site } \\
\hline Addis Ababa & $11(31.4)$ & $15(68.2)$ & $26(45.6)$ \\
\hline Amhara & $24(68.6)$ & $7(31.8)$ & $31(54.4)$ \\
\hline \multicolumn{4}{|c|}{ Disease on-set season } \\
\hline January-March & $7(20)$ & $6(27.3)$ & $13(22.8)$ \\
\hline April-June & 11(31.4) & $6(27.3)$ & $17(29.8)$ \\
\hline July-September & $9(25.7)$ & $6(27.3)$ & $15(26.3)$ \\
\hline October-December & $8(22.9)$ & $4(18.1)$ & $12(21.1)$ \\
\hline
\end{tabular}

$\mathrm{IPD}=$ Invasive pulmonary diseases, $\mathrm{N}=$ Number 
Almost all (75.4\%) isolates were resistant to at least one of the antibiotics tested. Intermediately resistant and fully resistant rates to penicillin were $26.3 \%$ and $17.5 \%$, respectively. The corresponding values for both cefotaxime and ceftriaxone were $3.5 \%$ and $1.8 \%$, respectively. Resistance rates to erythromycin, clindamycin, tetracycline, chloramphenicol and trimethoprim-sulfamethoxazole were $59.6 \%, 17.5 \%$, $38.6 \%, 17.5 \%$ and $24.6 \%$, respectively (Table 2 ). Norfloxacin (3.5\%) and oxacillin (38.6\%) were screening methods for the detection of moxifloxacin and levofloxacin, and penicillin-resistant pneumococci, respectively.

Table 2: Categorization of antimicrobial resistance to 10 antimicrobial agents in 57 Streptococcus pneumoniae isolates at Addis Ababa and Amhara region referral hospitals, Ethiopia, 2018-2019.

\begin{tabular}{|llll|}
\hline Antibiotics & Sensitive & Intermediate & Resistant \\
& $\mathbf{N}(\%)$ & $\mathbf{N}(\%)$ & $\mathbf{N}(\%)$ \\
\hline Penicillin G (PEN) & $32(56.1)$ & $15(26.3)$ & $10(17.5)$ \\
\hline Cefotaxime (CTX) & $54(94.7)$ & $2(3.5)$ & $1(1.8)$ \\
\hline Ceftriaxone (CRO) & $54(94.7)$ & $2(3.5)$ & $1(1.8)$ \\
\hline Erythromycin (ERY) & $23(40.4)$ & 0 & $34(59.6)$ \\
\hline Clindamycin (CD) & $46(80.7)$ & $1(1.8)$ & $10(17.5)$ \\
\hline Tetracycline (TET) & $33(57.9)$ & $2(3.5)$ & $22(38.6)$ \\
\hline Chloramphenicol (CHL) & $47(82.5)$ & 0 & $10(17.5)$ \\
\hline Trimethoprim-sulfamethoxazole (SXT) & $43(75.4)$ & 0 & $14(24.6)$ \\
\hline Norfloxacin (NOR) & $55(96.5)$ & NA & $2(3.5)$ \\
\hline Oxacillin (OXA) & $35(61.4)$ & NA & $22(38.6)$ \\
\hline
\end{tabular}

$N A=$ Not Applicable, $N=$ Number

Of the 57 isolates, 16 (28.1\%) were resistant to only one class of antibiotic, 14 (24.6\%) were resistant to two classes of antibiotics, while 19 (33.3\%) were resistant to three or more antibiotics; eight (14\%) isolates were susceptible to all antimicrobials tested. Penicillin, erythromycin, clindamycin and tetracycline-resistant pneumococcus were usually also resistant to other antibiotics. The most common MDR patterns were ERY+CD+TET (17.5\%), PEN+ERY+TET (10.5\%), followed by PEN+ERY+CD (7\%), PEN+CD+TET (7\%) and PEN+CTX+CRO+TET (7\%) (Table 3).

Table 3: Resistance to two or more antimicrobial agents among Streptococcus pneumoniae isolates at Addis Ababa and Amhara region referral hospitals, Ethiopia, 2018-2019. 


\begin{tabular}{|c|c|c|}
\hline Antibiotics & Number & Percentage \\
\hline PEN + ERY & 6 & 10.5 \\
\hline PEN + CD & 4 & 7 \\
\hline PEN + TET & 6 & 10.5 \\
\hline $\mathrm{PEN}+\mathrm{CHL}$ & 1 & 1.8 \\
\hline PEN + SXT & 3 & 5.3 \\
\hline$E R Y+C D$ & 10 & 17.5 \\
\hline ERY + TET & 19 & 33.3 \\
\hline $\mathrm{ERY}+\mathrm{CHL}$ & 9 & 15.8 \\
\hline$E R Y+S X T$ & 5 & 8.8 \\
\hline CD + TET & 10 & 17.5 \\
\hline$C D+S X T$ & 2 & 3.5 \\
\hline $\mathrm{TET}+\mathrm{CHL}$ & 1 & 1.8 \\
\hline TET + SXT & 5 & 8.8 \\
\hline $\mathrm{CHL}+\mathrm{SXT}$ & 1 & 1.8 \\
\hline PEN + CTX + CRO & 1 & 1.8 \\
\hline$P E N+E R Y+C D$ & 4 & 7 \\
\hline PEN + ERY + TET & 6 & 10.5 \\
\hline $\mathrm{PEN}+\mathrm{ERY}+\mathrm{CHL}$ & 1 & 1.8 \\
\hline $\mathrm{PEN}+\mathrm{ERY}+\mathrm{SXT}$ & 1 & 1.8 \\
\hline $\mathrm{PEN}+\mathrm{CD}+\mathrm{TET}$ & 4 & 7 \\
\hline$P E N+C D+S X T$ & 1 & 1.8 \\
\hline PEN + TET + CHL & 1 & 1.8 \\
\hline PEN + TET + SXT & 1 & 1.8 \\
\hline$E R Y+C D+T E T$ & 10 & 17.5 \\
\hline$E R Y+C D+S E T$ & 2 & 3.5 \\
\hline$C D+T E T+S X T$ & 2 & 3.5 \\
\hline $\mathrm{PEN}+\mathrm{CTX}+\mathrm{CRO}+\mathrm{ERY}$ & 1 & 1.8 \\
\hline PEN + CTX + CRO + TET & 4 & 7 \\
\hline
\end{tabular}




\begin{tabular}{|lll|}
\hline $\mathrm{PEN}+\mathrm{ERY}+\mathrm{CD}+\mathrm{SXT}$ & 1 & 1.8 \\
\hline $\mathrm{PEN}+\mathrm{CD}+\mathrm{TET}+\mathrm{SXT}$ & 1 & 1.8 \\
\hline $\mathrm{PEN}+\mathrm{ER}+\mathrm{ER}+\mathrm{CD}+\mathrm{TET}+\mathrm{SXT}$ & 1 & 1.8 \\
\hline $\mathrm{PEN}+\mathrm{CTE}+\mathrm{CRO}+\mathrm{ERY}+\mathrm{TET}+\mathrm{CHL}$ & 1 & 1.8 \\
\hline Total MDR (Greater than or equal to 3) & 19 & 33.3 \\
\hline
\end{tabular}

PEN- Penicillin, CTX- Cefotaxime, CRO- Ceftriaxone, ERY- Erythromycin, CD- Clindamycin, TETTetracycline, CHL-Chloramphenicol, SXT-Trimethoprim-sulfamethoxazole

\section{Discussion}

Streptococcus pneumoniae is responsible for many community infections, with the main ones being pneumonia and meningitis. Pneumococcus has developed increased resistance to multiple classes of antibiotics. Developing countries face significant problems of antimicrobial resistance with poor diagnostic facilities, unauthorized sale of antimicrobials, lack of appropriate functioning drug regulatory mechanisms, and non-human use of antimicrobials such as in animal production [13, 14]. In this work, we investigated the antimicrobial resistance profile and MDR pattern of $S$. pneumoniae from clinical isolates in two regions in Ethiopia. Currently, the antibiotic resistance patterns of $S$. pneumoniae isolates vary widely from one country to another, but many studies are reporting a high frequency of antimicrobial resistance among pneumococcal isolates.

In the present study, the resistance rate of pneumococci to a penicillin (17.5\%) was higher than other beta-lactam antibiotics such as cefotaxime (1.8\%) and ceftriaxone (1.8\%). Cefotaxime and ceftriaxone have a similar group of the spectrum. The resistance rate to penicillin in the present study was higher than studies in Central Africa (<6\%) [8], Tunisia (1.2\%) [13] and United States (14.8\%) [9], but higher penicillin resistance rates were observed in Taiwan (43.3-73.2\%) [14], Canada (26.1\%) [15], China (88.3\%) [16], Russia (28\%) [17], Nigeria (28\%) [18] and Guinea (21.5\%) [19]. The high prevalence of penicillin resistance in this report implies that the use of penicillin for empiric treatment of suspected pneumococcal infection should no longer be recommended. However, cefotaxime and ceftriaxone show very good activities against $S$. pneumoniae which might be due to their lack of availability and high cost compared to commonly used penicillin.

The overall resistance rate of $S$. pneumoniae to erythromycin, clindamycin, tetracycline, chloramphenicol, and trimethoprim/sulfamethoxazole was $59.6 \%, 17.5 \%, 38.6 \%, 17.5 \%$, and $24.6 \%$, respectively. The rate of resistance to erythromycin was 59.6\%. This was lower than in studies done in Canada (100\%) [15] and China (95.2\%) [16], but higher than in Russia (26\%) [17], Mozambique (23.6\%) [20] and Pakistan (up to 29.7\%) [21]. Seventeen and a half percent (17.5\%) isolates were resistant to clindamycin. Higher clindamycin resistance rates were observed in Canada (40.6\%) [15] and China (95.8\%) [16]. In addition, 
$38.6 \%$ of our isolates were resistant to tetracycline, which is lower than studies in China (93.6\%) [16] and Nigeria (73.5\%) [18]. Furthermore, the resistance rate to trimethoprim/sulfamethoxazole was $24.6 \%$ in our study. Higher resistance rates were reported in Central Africa (up to 69\%) [8], Canada (34.5\%) [15], China (66.7\%) [16], Russia (57\%) [17], Nigeria (96.2\%) [18] and Pakistan (86.6\%) [21]. In addition, 17.5\% of our strains were resistant to chloramphenicol, which is comparable to $18.9 \%$ in Central Africa [9], but lower than in Niger where up to $60 \%$ was reported [18].

These differences in antimicrobial resistance may be explained by differences in the source of the isolates, geographical variability, high prevalence of HIV infection, and antibiotics usage. Higher rates of antibiotic resistance have been linked to high antimicrobial consuming countries [10]. In Ethiopia, antibiotics can be bought without a prescription and this probably leads to overuse and misuse of antibiotics which can promote the widespread antibiotic resistance strain in the area [24].

The emergence of MDR S. pneumoniae isolates has been a worldwide public health concern for several years. In this study, we demonstrated that penicillin-resistant $S$. pneumoniae strains were also resistant to erythromycin, tetracycline, and clindamycin $(10.5 \%, 10.5 \%$, and $7 \%$ of isolates, respectively). Erythromycin resistant $S$. pneumoniae strains were resistant to tetracycline, clindamycin, and chloramphenicol $(33.3 \%$, $17.5 \%$, and $15.8 \%$ of isolates, respectively).

The most common MDR phenotype was resistance to penicillin, erythromycin, tetracycline, and clindamycin, detected in 33.3\% of the total isolates. Such MDR rates were higher than those observed in China (21.4\%) [16], Russia (22\%) [17] and Portugal (26\%) [22]. However, it was lower than studies reported from Tunisia (96.5\%) [13], Nigeria (53.8\%) [18] and China (99.4\%) [23]. Moreover, the results reveal a common pattern of co-resistance to penicillin, erythromycin, clindamycin, and tetracycline. The empirical treatment of pneumococcal infections, especially invasive infections, often requires a combination of two or more antibiotics and longer durations. These conditions inevitably lead to the initiation of high or multidrug resistance among patients with infections.

\section{Conclusions}

In the present study, most bacterial isolates were susceptible to ceftriaxone and cefotaxime. Penicillin has been used as a drug of choice for treating $S$. pneumoniae infection. However, antimicrobial resistance including multidrug resistance was observed to several commonly used antibiotics including penicillin. Hence, it is important to periodically monitor the antibiotic resistance patterns to choose empirical treatments for better management of pneumococcal infection. We propose to develop an action plan for the promotion of rational use of antimicrobials, strengthening of antimicrobial resistance surveillance, and implementation of an antimicrobial stewardship program

\section{Abbreviations}


CLSI: Clinical and Laboratory Standards Institute; CSF:Cerebrospinal Fluid; EUCAST:European Committee on Antimicrobial Susceptibility Testing; IPD:Invasive Pneumococcal Diseases; MDR:Multi-drug Resistance; MICs:Minimum Inhibitory Concentration; PCV:Pneumococcal Conjugate Vaccine; STGG:skimmed Milk-Trypticase Soy-Glucose-Glycerol; S. Pneumoniae:streptococcus Pneumoniae; WGS:Whole Genome Sequencing.

\section{Declarations}

\section{Acknowledgments}

The authors would like to thank the study participants. We are also very thankful for the institutional support grant from the University of Gondar. We are indebted to thank Microbiology laboratory staff of the hospitals and the University of Gondar for collecting and rechecking the isolates. We also would like to thank staffs from the Norwegian Institute of Public Health Microbiology Laboratory (specially Prof. Dominique A Caugant) for their assistance in facilitating the whole genome sequencing and drug resistance procedures.

\section{Authors' contributions}

BS, FM, GY, and BT conceived and designed the study. BS, WA, and SF carried out data collection. BS performed all microbiological analyses, under the supervision of DV. BS and DV analyzed the data and carried out the interpretation of the data. BS drafted the manuscript. BT, FM, GY, and DV critically revised the manuscript for intellectual content. All authors read and approved the final manuscript

\section{Funding}

This study was supported by Research and Community Services of University of Gondar with Grant No.VP/RCS/05/2/43/2017 and University of Gondar PhD researchgrant.

\section{Availability of data and materials}

All data generated or analyzed during this study are included in this published article.

\section{Consent for publication}

Not applicable.

\section{Competing interests}

The authors declare that they have no competing interests.

\section{Ethics approval and consent to participate}

The study was reviewed and approved by the Ethical Review Board of the University of Gondar, (No.O/V/P/RCS/05/377/2017). Permission was obtained from each hospital laboratories for collecting 
the isolates.

\section{Authors' Information}

1,2Bekele Sharew is a PhD student in Medical Microbiology, University of Gondar. Wollo University is his home institution. ${ }^{1}$ Belay Tessema (MSc., PhD), ${ }^{1}$ Feleke Moges (MSc., PhD., Professor), ${ }^{1}$ Gizachew Yismaw (MSc., PhD), and ${ }^{1}$ Wondiwossen Abebe (MSc) are at College of Medicine and Health Sciences, Department of Medical Microbiology, University of Gondar. ${ }^{3}$ Surafal Fentaw (MSc) is at Ethiopia Public Health Institute, Addis Ababa. and ${ }^{4}$ Didrik Vestrheim (MD; PhD) are at Norwegian Institute of Public Health, Oslo, Norway.

\section{References}

1. Wahl B, O’Brien KL, Greenbaum A, Majumder A, Liu L, Chu Y, Lukšić I, Nair H, McAllister DA,Campbell H, Rudan I, Black R and Knoll MD. Burden of Streptococcus pneumoniae and Haemophilus influenzae type $b$ disease in children in the era of conjugate vaccines: global, regional, and national estimates for 2000-15. Lancet Glob Health. 2018; 6: e744-57

2. Parks $T$, Barrett $L$ and Jones $N$. Invasive streptococcal disease: A review for clinicians. British Medical Bulletin. 2015; 115(1):77- Doi:10.1093

3. Jacobs MR. Streptococcus pneumoniae: Epidemiology and Patterns of Resistance. Am J Med. 2004;117(3): 3-15. Doi:10.1016

4. Karchmer AW. Increased Antibiotic Resistance in Respiratory Tract Pathogens: PROTEKE US-An update. Clin Infect Dis. 2004;39 (3S): S142-S150. Doi:10.1086/421352.

5. Jean S.-S and Hsueh P.-R. High burden of antimicrobial resistance in Asia. International Journal of Antimicrobial Agents. 2011; (37):291-295.

6. Hsueh PR and Luh KT. Antimicrobial Resistance in Streptococcus pneumoniae, Emerging Infectious Diseases. 2002; 8:12.

7. Siira L, Jalava J, Tissari P, Vaara M, Kaijalainen T and Virolainen A. Clonality behind the increase of multidrug-resistanceamong non-invasive pneumococci in Southern Finland. Eur J Clin Microbiol Infect Dis. 2012; 31:867-

8. Vlieghe E, Phoba M.F, Muyembe Tamfun JJ and Jacobs J.Antibiotic resistance among bacterial pathogens in Central Africa: a reviewof the published literature between 1955 and 2008. International Journal of Antimicrobial Agents. 2009; (34):295-303.

9. Jones RN, Sader HS, Mendes RE, and Flamm RK. Update on antimicrobial susceptibility trends among Streptococcus pneumoniae in theUnited States: report of ceftaroline activity from the SENTRY AntimicrobialSurveillance Program (1998-2011). Diagnostic Microbiology and Infectious Diseases. 2013; (75):107-109.

10. Goossens H, Ferech M, Vander Stichele R and Elseviers M, for the ESAC Project Group. Outpatient antibiotic use in Europe and association withresistance: a cross-national database study. Lancet. 
2005; 365: 579-587

11. Wayne PA. Performance Standards for Antimicrobial Susceptibility Testing. $30^{\text {th }}$ CLSI supplement M100. CLSI; 2020.

12. The European Committee on Antimicrobial Susceptibility Testing (EUCAST). Breakpoint tables for interpretation of MICs and zone diameters. Version 10.0, 2020. http://www.eucast.org.

13. Byarugaba DK. A view on antimicrobial resistance in developing countries andresponsible risk factors. Int J Antimicrob Agents. 2004; 24:105-110. kh

14. Ayukekbong JA, Ntemgwa M \& Atabe The threat of antimicrobial resistance in developing countries: causes and control strategies. Antimicrobial Resistance and Infection Control. 2017; 6:47.

15. Midouni Ayadi B, Mehiri E, Draoui H, Ghariani A, Essalah L, Raoult D, Fournier PE and Slim-Saidi LN. Phenotypic and molecular characterization of macrolideresistance mechanisms among Streptococcus pneumoniaeisolated in Tunisia. Journal of Medical Microbiology. 2020; 69:505-520

16. Li C-F, Liu M-F, Shi Z-Y,Hsueh P-R, Liao C-H, Jang T-N,Tsao S-M, Kung H-C, Hsu G-J, Cheng Y-J, Lin HC, Liu Y-C,Chuang Y-C, Wang L-S and Chen C-M. Changing trends in antimicrobial susceptibility ofStreptococcus pneumoniae isolates in Taiwan. Journal of Microbiology, Immunology and Infectious. 2012; 45:305-310. 2006e2007

17. Wierzbowski AK, Karlowsky JA, Adam HJ, Nichol KA, Hoban DJand Zhanel GG, on behalf of the Canadian Antimicrobial Resistance Alliance (CARA). Evolution and molecular characterization of macrolide-resistantStreptococcus pneumoniae in Canada between 1998 and 2008. Antimicrob Chemother. 2014; 69:59-66. doi:10.1093/jac/dkt332.

18. Wang C, Chen Y-H, Fang C, Zhou M, Xu H, Jing C, Deng H, Cai H, Jia K, Han S, Yu H, Wang A, Yin D, Wang C, Wang W, Huang W, Deng J, Zhao R, Chen Y, Yang J-H, Wang C, Che $Y$, Nie $X$, Wang $S$, Hao $J$ and Zhang $C$. Antibiotic resistance profiles and multidrug resistance patterns of Streptococcus pneumoniae in pediatrics. A multicenter retrospective study in mainland China. Medicine. 2019; 98:24(e15942)

19. Mayanskiy N, Alyabieva N, Ponomarenko O, Lazareva A,Katosova L, Ivanenko A, Kulichenko T,Namazova-Baranova $L$ and Baranov A. Serotypes and antibiotic resistance of non-invasive Streptococcuspneumoniae circulating in pediatric hospitals in Moscow, Russia. International journal of Infectious diseases. 2014; 20:58-62.

20. Iliyasu G, Habib AG and Aminu MB. Antimicrobialsusceptibility pattern of invasive pneumococcal isolates in North West J Global Infect Dis. 2015; 7:70-74.

21. Greenhill AR, Phuanukoonnon S, Michael A, Yoannes M, Orami T, Smith $H$, Murphy D, Blyth C, Reeder J, Siba P, Pomat W and Lehmann D. Streptococcus pneumoniae and Haemophilus influenzae in paediatric meningitis patients at Goroka General Hospital, Papua New Guinea: serotype distribution and antimicrobial susceptibility in the pre-vaccine era. BMC Infectious Diseases. 2015; 15:485. DOI 10.1186/s12879-015-1197-0

22. Bos JC, Beishuizen SJ, Madeira GC, dos Santos Gomonda E, Cossa EO, Macome AC, van Steenwijk RP, Schultsz C and Prins JM Antimicrobial susceptibility of 
Streptococcus pneumoniae in adult patients with pneumococcal pneumonia in an urban hospital in Mozambique. BMC Research Notes. 2014; 7:110.

23. Zafar A, Hasan R, Nizamuddin S, Mahmood N, Mukhtar S, Ali F, Morrissey I, Barker K and Torumkuney D. Antibiotic susceptibility in Streptococcus pneumoniae, Haemophilus influenzae and Streptococcus pyogens in Pakistan: a review of results from the Survey of Antibiotic Resistance (SOAR) 2002-15. J Antimicrob Chemother. 2016; 71 Suppl 1: i103-i109. doi:10.1093/jac/dkw076

24. Abera B, Kibret M and Mulu W. Knowledge and beliefs on antimicrobial resistance among physicians and nurses in hospitals in Amhara Region, Ethiopia. BMC Pharmacology and Toxicology. 2014, $15: 26$

25. Nunes S, Sa-Leao R, Carrico J, Alves CR, Mato R, Avo AB, Saldanha J,Almeida JS, Sanches IS, and de Lencastre H. Trends in Drug Resistance, Serotypes, and Molecular Types of Streptococcus pneumoniae Colonizing Preschool-Age ChildrenAttending Day Care Centers in Lisbon, Portugal: A Summary of 4 Years of Annual Surveillance. Journal of Clinical Microbiology. 2005; 1285-1293.

26. Geng Q, Zhang T, Ding Y, Tao Y, Lin Y, Wang Y, Black S and Zhao G. Molecular Characterization and Antimicrobial Susceptibility of Streptococcus pneumoniae Isolated from Children Hospitalized with Respiratory Infections in Suzhou, China. PLOS ONE. 2014. 9(4): e93752. doi: 10.1371/journal.pone.0093752 